\title{
COMPLETION OF THE CATEGORY OF FINITE-DIMENSIONAL FUZZY SPACES
}

\author{
NGUYEN NHUY, PHAM QUANG TRINH \\ and \\ VU THI HONG THANH
}

\begin{abstract}
In this paper we introduce a method to expand the category $\mathcal{F}$ of all finite-dimensional fuzzy spaces associated with finite-dimensional Chu spaces into a complete system.

Tóm tăt. Bài này tiếp tục nghiên cúu phạm trù các không gian’ mờ hữu hạn chiều đã được đề cập đến trong [7] và [8]. Như dã dược chứng minh trong [7], phạm trù $\mathcal{\exists}$ các không gian mò̀ hũu hạn chiều liên kết với các không gian Chu hũu hạn chiều là một hệ thống tương đương, tuy nhiên, $\mathcal{F}$ không đóng đối với phép lấy tích chéo nên nó không là một hệ thống đầy đư. Trong bài này, chúng tôi đưa ra một phương pháp mở rộng phạm trù $\mathcal{F}$ thành một hệ thống đầy đư. Để làm điều đó, chúng tôi xây dựng một phạm trù $n$-tập hợp đối ngẫu $\mathcal{F}^{*}$ chứa $\mathcal{F}$ nhu một phạm trù con, trong đó $\mathcal{F}^{*}$ là một hệ thống đầy đú.
\end{abstract}

\section{INTRODUCTION}

It is shows in [7] that, the category $\mathcal{F}$ of all finite-dimensional fuzzy spaces associated with finite-dimensional Chu spaces is an equivalent system. Unfortunately, $\mathcal{F}$ is not closed under the cross product, therefore $\mathcal{F}$ is not a complete system. In this paper we introduce a method to expand the category $\mathcal{F}$ into a complete system, that is, we construct a "dual" $n$-set category $\mathcal{F}^{*}$ containing $\mathcal{F}$ as a subcategory, where $\mathcal{F}^{*}$ is a complete system.

\section{FINITE-DIMENSIONAL *-FUZZY SPACES AND THE *-FUZZY FUNCTOR}

By $n$-set we mean a cartesian product $X=\prod_{i=1}^{n} X_{i}$. Let $S$ denote the $n$-set category, when the category $S^{*}$ is defined as follows:

1. Objects of $S^{*}$ are morphisms in $S$.

2. If $\alpha: X=\prod_{i=1}^{n} X_{i} \rightarrow Y=\prod_{i=1}^{n} Y_{i}$ and $\alpha^{\prime}: X^{\prime}=\prod_{i=1}^{n} X_{i}^{\prime} \rightarrow Y^{\prime}=\prod_{i=1}^{n} Y_{i}^{\prime}$ are two objects of $S^{*}$, then a morphism $\varphi: \alpha \rightarrow \alpha^{\prime}$ from $\alpha$ to $\alpha^{\prime}$ in $S^{*}$ is a map (in the $n$-set category) $\varphi: Y=\prod_{i=1}^{n} Y_{i} \rightarrow X^{\prime}=\prod_{i=1}^{n} X_{i}^{\prime}$.

Let $\alpha: X=\prod_{i=1}^{n} X_{i} \rightarrow Y=\prod_{i=1}^{n} Y_{i}, \alpha^{\prime}: X^{\prime}=\prod_{i=1}^{n} X_{i}^{\prime} \rightarrow Y^{\prime}=\prod_{i=1}^{n} Y_{i}^{\prime}$ and $\alpha^{\prime \prime}: X^{\prime \prime}=$ $\prod_{i=1}^{n} X_{i}^{\prime \prime} \rightarrow Y^{\prime \prime}=\prod_{i=1}^{n} Y_{i}^{\prime \prime}$ be objects in $S^{*}, \varphi: \alpha \rightarrow \alpha^{\prime}$ and $\varphi^{\prime}: \alpha^{\prime} \rightarrow \alpha^{\prime \prime}$ be morphisms of $\mathcal{S}^{*}$ (i.e., $\varphi: Y=\prod_{i=1}^{n} Y_{i} \rightarrow X^{\prime}=\prod_{i=1}^{n} X_{i}^{\prime}$ and $\left.\varphi^{\prime}: Y^{\prime}=\prod_{i=1}^{n} Y_{i}^{\prime} \rightarrow X^{\prime \prime}=\prod_{i=1}^{n} X_{i}^{\prime \prime}\right)$.

Then composition of $\varphi$ and $\varphi^{\prime}$, denoted by $\varphi^{\prime} * \varphi$, is given by

$$
\varphi^{\prime} * \varphi=\varphi^{\prime} \alpha^{\prime} \varphi: \alpha \rightarrow \alpha^{\prime \prime} .
$$

It is easy to check that with the above definition $S^{*}$ is a category.

For a given set $X=\prod_{i=1}^{n} X_{i}$, let $X^{*}=[0,1]^{X}$ denote collection of all fuzzy sets of X. For a map $\alpha: X=\prod_{i=1}^{n} X_{i} \rightarrow Y=\prod_{i=1}^{n} Y_{i}$ we define the conjugate $\alpha^{*}: Y^{*} \rightarrow X^{*}$ of $\alpha$ by the formula

$$
\alpha^{*}(a)(x)=a(\alpha(x)) \text { for } x \in X \text { and } a \in Y^{*} .
$$

It is easy to see that

$$
(\beta \alpha)^{*}=\alpha^{*} \beta^{*} \text { for every } \alpha: X \rightarrow Y \text { and } \beta: Y \rightarrow Z .
$$


Now for $\alpha: X=\prod_{i=1}^{n} X_{i} \rightarrow Y=\prod_{i=1}^{n} Y_{i}$ we define $F^{*}(\alpha)=\left(\prod_{i=1}^{n} X_{i}, f_{\alpha}, Y^{*}\right)$, where $Y^{*}$ denotes the collection of all fuzzy sets of $Y=\prod_{i=1}^{n} Y_{i}$, and $f_{\alpha}: \prod_{i=1}^{n} X_{i} \times Y^{*} \rightarrow[0,1]$ is given by

$$
f_{\alpha}\left(x_{1}, x_{2}, \ldots, x_{n}, a\right)=a\left(\alpha\left(x_{1}, x_{2}, \ldots, x_{n}\right)\right) \text { for every }\left(x_{1}, x_{2}, \ldots, x_{n}, a\right) \in \prod_{i=1}^{n} X_{i} \times Y^{*} .
$$

The $(n+1)$-dimensional Chu space $F^{*}(\alpha)=\left(\prod_{i=1}^{n} X_{i}, f_{\alpha}, Y^{*}\right)$ is called the $(n+1)$-dimensional ${ }^{*}$-fuzzy space associated with the map $\alpha: X=\prod_{i=1}^{n} X_{i} \rightarrow Y=\prod_{i=1}^{n} Y_{i}$. The category of all $(n+1)$-dimensional *-fuzzy spaces associated with maps in the $n$-set category $S$ is called the $(n+1)$ dimensional ${ }^{*}$-fuzzy category and denoted by $\mathcal{F}^{*}$.

\section{RESULTS}

At first, we will show that the $(n+1)$-dimensional *-fuzzy category $\mathcal{F}^{*}$ defined above contains the category $\mathcal{F}$ as a subcategory. In fact, we have the following theorem.

Theorem 1. Any $(n+1)$-dimensional fuzzy space is a $(n+1)$-dimensional ${ }^{*}$-fuzzy space.

Proof. If $F(X)=\left(\prod_{i=1}^{n} X_{i}, f_{X^{*}}, X^{*}\right)$ then clearly that $F(X)=F^{*}\left(1_{X}\right)$ is a $(n+1)$-dimensional *-fuzzy space.

Theorem 2. $\mathcal{F}^{*}$ is a complete system.

Proof. Assume that $\Phi=\left(\prod_{i=1}^{n} \varphi_{i}, \psi\right): F^{*}(\alpha)=\left(\prod_{i=1}^{n} X_{i}, f_{\alpha}, Y^{*}\right) \rightarrow F^{*}\left(\alpha^{\prime}\right)=\left(\prod_{i=1}^{n} X_{i}^{\prime}, f_{\alpha^{\prime}}, Y^{\prime *}\right)$ is a $(n+1)$-Chu morphism, where $F^{*}(\alpha)$ and $F^{*}\left(\alpha^{\prime}\right)$ are $(n+1)$-dimensional ${ }^{*}$-fuzzy spaces associated with the maps $\alpha=\prod_{i=1}^{n} \alpha_{i}: X=\prod_{i=1}^{n} X_{i} \rightarrow Y=\prod_{i=1}^{n} Y_{i}$ and $\alpha^{\prime}=\prod_{i=1}^{n} \alpha_{i}^{\prime}: X^{\prime}=\prod_{i=1}^{n} X_{i}^{\prime} \rightarrow$ $Y^{\prime}=\prod_{i=1}^{n} Y_{i}^{\prime}$, respectively. Putting $\beta=\alpha^{\prime} \varphi=\prod_{i=1}^{n} \alpha_{i}^{\prime} \varphi_{i}: X=\prod_{i=1}^{n} X_{i} \rightarrow Y^{\prime}=\prod_{i=1}^{n} Y_{i}^{\prime}$, we get the cross product $\tilde{C}=\left(\prod_{i=1}^{n} X_{i}, f_{\alpha} \times_{\Phi} f_{\alpha^{\prime}}, Y^{\prime *}\right)$, which is a $(n+1)$-dimensional ${ }^{*}$-fuzzy space associated with the map $\beta=\prod_{i=1}^{n} \alpha_{i}^{\prime} \varphi_{i}$. In fact, for every $\left(x_{1}, \ldots, x_{n}, b\right) \in \prod_{i=1}^{n} X_{i} \times Y^{\prime *}$, we have

$$
\begin{aligned}
\left(f_{\alpha} \times_{\Phi} f_{\alpha^{\prime}}\right)\left(x_{1}, \ldots, x_{n}, b\right) & =f_{\alpha^{\prime}}\left(\varphi_{1}\left(x_{1}\right), \ldots, \varphi_{n}\left(x_{n}\right), b\right) \\
& =b\left(\alpha_{1}^{\prime} \varphi_{1}\left(x_{1}\right), \ldots, \alpha_{n}^{\prime} \varphi_{n}\left(x_{n}\right)\right) \\
& =f_{\alpha^{\prime} \varphi}\left(x_{1}, \ldots, x_{n}, b\right) \\
& =f_{\beta}\left(x_{1}, \ldots, x_{n}, b\right) .
\end{aligned}
$$

Thus, the category $\mathcal{F}^{*}$ is closed under the cross product. Therefore the theorem is proved.

Theorem 3. $F^{*}: S^{*} \rightarrow \mathcal{F}^{*}$ is a covariant functor.

Proof. For a morphism $\varphi=\prod_{i=1}^{n} \varphi_{i}: \alpha=\prod_{i=1}^{n} \alpha_{i} \rightarrow \alpha^{\prime}=\prod_{i=1}^{n} \alpha_{i}^{\prime}$, with $\alpha, \alpha^{\prime} \in S^{*}$, we define

$$
F^{*}(\varphi)=\left(\prod_{i=1}^{n} \varphi_{i} \alpha_{i}, \varphi^{*} \alpha^{*}\right)
$$

where $\varphi^{*}$ and $\alpha^{\prime *}$ are conjugated of $\varphi=\prod_{i=1}^{n} \varphi_{i}$ and $\alpha^{\prime}=\prod_{i=1}^{n} \alpha_{i}^{\prime}$, respectively, that is

$$
\varphi^{*}(a)\left(y_{1}, \ldots, y_{n}\right)=a\left(\varphi_{1}\left(y_{1}\right), \ldots, \varphi_{n}\left(y_{n}\right)\right) \text { for every }\left(y_{1}, \ldots, y_{n}\right) \in \prod_{i=1}^{n} Y_{i} \text { and } a \in X^{\prime *}
$$

and

$$
\alpha^{\prime *}(b)\left(x_{1}^{\prime}, \ldots, x_{n}^{\prime}\right)=b\left(\alpha_{1}^{\prime}\left(x_{1}^{\prime}\right), \ldots, \alpha_{n}^{\prime}\left(x_{n}^{\prime}\right)\right) \text { for every }\left(x_{1}^{\prime}, \ldots, x_{n}^{\prime}\right) \in \prod_{i=1}^{n} X_{i}^{\prime} \text { and } b \in Y^{\prime *} \text {. }
$$

We claim that $F^{*}(\varphi): F^{*}(\alpha)=\left(\prod_{i=1}^{n} X_{i}, f_{\alpha}, Y^{*}\right) \rightarrow F^{*}\left(\alpha^{\prime}\right)=\left(\prod_{i=1}^{n} X_{i}^{\prime}, f_{\alpha^{\prime}}, Y^{\prime *}\right)$ is a $(n+1)$ dimensional Chu morphism. That is, the following diagram commutes: 


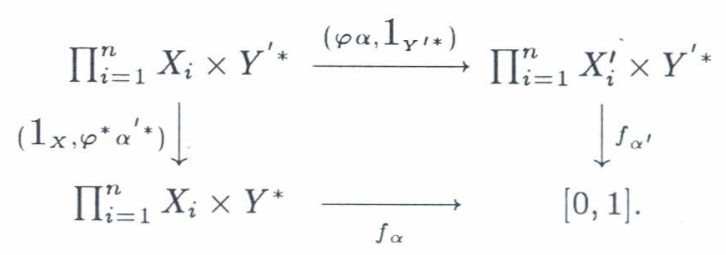

In fact, for every $\left(x_{1}, \ldots, x_{n}\right) \in \prod_{i=1}^{n} X_{i}$ and $b \in Y^{\prime *}$, we have

$$
\begin{aligned}
f_{\alpha}\left(x_{1}, \ldots, x_{n}, \varphi^{*} \alpha^{\prime *}(b)\right) & =\varphi^{*} \alpha^{\prime *}(b)\left(\alpha_{1}\left(x_{1}\right), \ldots, \alpha_{n}\left(x_{n}\right)\right) \\
& =\left(\alpha^{\prime} \varphi\right)^{*}(b)\left(\alpha_{1}\left(x_{1}\right), \ldots, \alpha_{n}\left(x_{n}\right)\right) \\
& =b\left(\alpha_{1}^{\prime} \varphi_{1} \alpha_{1}\left(x_{1}\right), \ldots, \alpha_{n}^{\prime} \varphi_{n} \alpha_{n}\left(x_{n}\right)\right) \\
& =f_{\alpha^{\prime}}(\varphi \alpha(x), b)
\end{aligned}
$$

Consequently the above diagram commutes.

Hence $F^{*}(\varphi)=\left(\prod_{i=1}^{n} \varphi_{i} \alpha_{i}, \varphi^{*} \alpha^{*}\right)$ is a $(n+1)$-Chu morphism.

Now we will show that $F^{*}$ preserves the composition. In fact, let

$$
\alpha=\prod_{i=1}^{n} \alpha_{i}: X=\prod_{n=1}^{n} X_{i} \rightarrow Y=\prod_{i=1}^{n} Y_{i}, \quad \alpha^{\prime}=\prod_{i=1}^{n} \alpha_{i}^{\prime}: X^{\prime}=\prod_{i=1}^{n} X_{i}^{\prime} \rightarrow Y^{\prime}=\prod_{i=1}^{n} Y_{i}^{\prime}
$$

and

$$
\alpha^{\prime \prime}=\prod_{i=1}^{n} \alpha_{i}^{\prime \prime}: X^{\prime \prime}=\prod_{i=1}^{n} X_{i}^{\prime \prime} \rightarrow Y^{\prime \prime}=\prod_{i=1}^{n} Y_{i}^{\prime \prime}
$$

be objects in the category $S^{*}$. Let $\varphi=\prod_{i=1}^{n} \varphi_{i}: \alpha=\prod_{i=1}^{n} \alpha_{i} \rightarrow \alpha^{\prime}=\prod_{i=1}^{n} \alpha_{i}^{\prime}$ and $\varphi^{\prime}=\prod_{i=1}^{n} \varphi_{i}^{\prime}$ : $\alpha^{\prime}=\prod_{i=1}^{n} \alpha_{i}^{\prime} \rightarrow \alpha^{\prime \prime}=\prod_{i=1}^{n} \alpha_{i}^{\prime \prime}$ be morphisms in $S^{*}$ (i.e., $\varphi=\prod_{i=1}^{n} \varphi_{i}: Y=\prod_{i=1}^{n} Y_{i} \rightarrow X^{\prime}=$ $\prod_{i=1}^{n} X_{i}^{\prime}$ and $\varphi^{\prime}=\prod_{i=1}^{n} \varphi_{i}^{\prime}: Y^{\prime}=\prod_{i=1}^{n} Y_{i}^{\prime} \rightarrow X^{\prime \prime}=\prod_{i=1}^{n} X_{i}^{\prime \prime}$ are maps in the n-set category). By the definition we have $\varphi^{\prime} * \varphi=\varphi^{\prime} \alpha^{\prime} \varphi=\prod_{i=1}^{n} \varphi_{i}^{\prime} \alpha_{i}^{\prime} \varphi_{i}$. Therefore

$$
\begin{aligned}
F^{*}\left(\varphi^{\prime} * \varphi\right) & =\left(\varphi^{\prime} \alpha^{\prime} \varphi \alpha,\left(\varphi^{\prime} \alpha^{\prime} \varphi\right)^{*} \alpha^{\prime \prime}\right) \\
& =\left(\varphi^{\prime} \alpha^{\prime} \varphi \alpha, \varphi^{*} \alpha^{\prime *} \varphi^{\prime *} \alpha^{\prime \prime}\right) \\
& =F^{*}\left(\varphi^{\prime}\right) F^{*}(\varphi) .
\end{aligned}
$$

Consequently $F^{*}$ preserves the composition, and hence $F^{*}: S^{*} \rightarrow \mathcal{F}^{*}$ is a covariant functor.

The functor $F^{*}: S^{*} \rightarrow \mathcal{F}^{*}$ is called $(n+1)$-dimensional ${ }^{*}$-fuzzy functor.

Acknowledgement. The authors are thankful to N.T. Hung of New Mexico for his comments during the preparation of this paper.

\section{REFERENCES}

[1] Barr M., * -Autonomous categories, Lecture Notes in Mathematics, \#752, Springer-Verlag; Electronic Notes in Theorestical Computer Science, 1979.

[2] Barry Mitchell, Theory of Categories, New York and London, 1965.

[3] Barwise J. and Seligman J., Information Flow, The Logic of Distributed Systems, Cambridge Univ. Pess, 1977.

[4] Gupta V., "Chu spaces: a model of concurrency", Ph.D.thesis, Stanford Univ., Available at ftp:// boole.stanford.edu/pub/gupthes.ps.Z., 1994.

[5] Nguyen H. T. and Walker E., A First Course in Fuzzy Logic, Boca Raton, FL: CRC, 1997 (2nd ed., 1999).

[6] Nguyen H. T. and Sugeno M., Fuzzy Systems: Modeling and Control, Kluwer Academic, 1998. 
[7] Nguyen Nhuy, Pham Quang Trinh, and Vu Hong Thanh, Finite-dinesional Chu space, Journal of Computer Science and Cybernetics 15 (4) (1999).

[8] Nguyen Nhuy and Vu Hong Thanh, Finite-dimensional Chu space, Fuzzy space and the game Invariance Theorem, to apper in Journal of Computer Science and Cybernetics.

[9] Paradopoulos B. K. and Syropoulos A., Fuzzy sets and fuzzy relational structures as Chu spaces, Proceedings of the First International Workshop on Current Trends and Developments of Fuzzy Logic, Thessaloniki, Greece, Oct. 16-20, 1998; Electronic Notes in Theoretical Computer Science (1998).

[10] Pratt V.R., Type as procsses, via Chu spaces, Electronic Notes in Theoretical Computer Science 7 (1997).

[11] Pratt V.R., Chu spaces as a sematic bridge between linear logic and mathematics, Electronic Notes in Theoretical Computer Science 12 (1998).

Department of Information Technology,

Received August 11, 2000

Vinh University, Nghe An, Vietnam. 